\title{
An Adaptive Strategy for Active Debris Removal
}

\author{
Adam E. White ${ }^{\mathrm{a}^{*}},{ }_{\text {Hugh G. Lewis }}{ }^{\mathrm{a}}$ \\ ${ }^{a}$ Astronautics Research Group, Faculty of Engineering and the Environment, University of \\ Southampton, Southampton, SO17 1BJ, United Kingdom
}

\begin{abstract}
Many parameters influence the evolution of the near-Earth debris population, including launch, solar, explosion and mitigation activities, as well as other future uncertainties such as advances in space technology or changes in social and economic drivers that effect the utilisation of space activities. These factors lead to uncertainty in the long-term debris population. This uncertainty makes it difficult to identify potential remediation strategies, involving active debris removal (ADR), that will perform effectively in all possible future cases. Strategies that cannot perform effectively, because of this uncertainty, risk either not achieving their intended purpose, or becoming a hindrance to the efforts of spacecraft manufactures and operators to address the challenges posed by space debris.

One method to tackle this uncertainty is to create a strategy that can adapt and respond to the space debris population. This work explores the concept of an adaptive strategy, in terms of the number of objects required to be removed by ADR, to prevent the low Earth orbit (LEO) debris population from growing in size. This was demonstrated by utilising the University of Southampton's Debris Analysis and Monitoring Architecture to the Geosynchronous Environment (DAMAGE) tool to investigate ADR rates (number of removals per year) that change over time in response to the current space environment, with the requirement of achieving zero growth of the LEO population.

DAMAGE was used to generate multiple Monte Carlo projections of the future LEO debris environment. Within each future projection, the debris removal rate was derived at five-year intervals, by a new statistical debris evolutionary model called the Computational Adaptive Strategy to Control Accurately the Debris Environment (CASCADE) model. CASCADE predicted the long-term evolution of the current DAMAGE population with a variety of different ADR rates in order to identify a removal rate that produced a zero net growth for that particular projection after 200 years.

The results show that using an adaptive ADR rate generated by CASCADE, alongside good compliance with existing mitigation measures, increases the probability of achieving a constant LEO population of objects greater than $10 \mathrm{~cm}$. This was shown to be 12 per cent greater compared with removing five objects per year, with the additional advantage of requiring only 3.1 removals per year, on average.
\end{abstract}

*Corresponding author email: adam.white@southampton.ac.uk, Tel: +44 2380597658 


\section{Nomenclature}

\begin{tabular}{|c|c|}
\hline$\dot{C}$ & Intact object collision rate (\#/year) \\
\hline$C N_{R}$ & Cumulative number of objects removed in an ADR scenario \\
\hline$\dot{D} c, \dot{D} e, \dot{D} i$ & Drag rates for collision, explosion and intact objects (\#/year) \\
\hline$\dot{E}$ & Explosion rate (\#/year) \\
\hline$F$ & A scaling factor implemented within the NASA standard breakup model \\
\hline$h$ & Altitude band number \\
\hline$k_{1} \ldots k_{4}$ & Collisions coefficients \\
\hline$\dot{L}$ & Launch rate (\#/year) \\
\hline$L_{c}$ & The minimum characteristic length of a fragment $(\mathrm{m})$ \\
\hline$m_{\text {avg }}$ & The average mass of an intact rocket body or satellite $\geq 10 \mathrm{~cm}$ in size $(\mathrm{kg})$ \\
\hline$m_{i}$ & Mass of an intact object $i(\mathrm{~kg})$ \\
\hline$N$ & Number of objects $\geq 10 \mathrm{~cm}$ in CASCADE \\
\hline$N_{A}$ & Number of objects $\geq 10 \mathrm{~cm}$ in a scenario with no ADR \\
\hline$N_{B}$ & Number of objects $\geq 10 \mathrm{~cm}$ in an ADR scenario \\
\hline $\mathrm{Nc}, \mathrm{Ne}, \mathrm{Ni}$ & Number of objects $\geq 10 \mathrm{~cm}$ for collision, explosion and intact objects \\
\hline$N_{D}$ & Number of objects $\geq 10 \mathrm{~cm}$ in a DAMAGE projection \\
\hline$N_{p}$ & Growth of the CASCADE population compared to the initial DAMAGE population \\
\hline$n_{e}$ & Number of explosion fragments generated per explosion \\
\hline$n_{c}$ & Number of explosion fragments generated per collision \\
\hline$\dot{P}$ & PMD compliance rate (\#/year) \\
\hline$P_{i}$ & Collision probability of object $i$ \\
\hline$R^{2}$ & Coefficient of determination \\
\hline$\dot{R}$ & Rate of objects removed by ADR (\#/year) \\
\hline$S$ & Scaling factor to replicate the effect of solar activity on atmospheric drag \\
\hline$t$ & Current projection time (year) \\
\hline$t_{e}$ & Time at end of a projection (year) \\
\hline$T_{i}$ & DAMAGE target selection criterion for ADR \\
\hline$t_{0}$ & Time at beginning of a projection (year) \\
\hline$\Delta t$ & Time step (days) \\
\hline
\end{tabular}




\section{Introduction}

Space debris is a threat to the safe operation of near-Earth satellites and the long-term sustainability of outer space activities. In the last decade, modelling studies, such as Liou and Johnson (2006), have predicted that the current debris population in low Earth orbit (LEO) has reached a sufficient density at some altitudes for collision activity there to continue even in the absence of any new launches.

Indeed, in 2009, the Inter-Agency Space Debris Coordination Committee (IADC) initiated an Action Item (AI 27.1) to determine the stability of the future LEO space debris environment (IADC, 2013); and as such establish whether measures such as active debris removal (ADR) should be investigated further. In doing so, optimistic levels of mitigation (90\% future level of compliance with a 25-year post-mission disposal (PMD) rule and 100\% passivation of satellites and rocket bodies) and potentially optimistic levels in launch and solar activity were used. Despite these optimistic values, using six agencies' modelling tools, a consensus was reached that confirmed the current $\geq 10 \mathrm{~cm}$ LEO debris population was still likely to grow. A key conclusion of the report was that:

“...to stabilize the LEO environment, more aggressive measures, such as active debris removal, should be considered."

(IADC report, Stability of the Future LEO Environment, IADC-12-08, Rev. 1, page 17, Jan 2013)

Recent ADR modelling studies, completed by the International Academy of Astronautics (Klinkrad and Johnson, 2010), the National Aeronautics and Space Administration's (NASA's) Orbital Debris Program Office (Liou et al., 2010), the University of Southampton (Lewis et al., 2012), and others have all demonstrated the effect of ADR. Results have shown that it may be possible to prevent the expected growth of the $\geq 10 \mathrm{~cm}$ LEO population by removing in the order of three (Lewis et al., 2012) to 15 (Klinkrad and Johnson, 2010) well-chosen debris objects per year alongside widespread compliance with IADC Space Debris Mitigation Guidelines (IADC, 2007).

These ADR studies have taken some reasonable, although arguably optimistic assumptions, concerning the future, that constrain parameters such as launch, explosion, solar and mitigation activity, to a limited number of cases. As a result, amongst other reasons, current removal rates are proposed only to serve as a guide for the further development of high-level ADR requirements. J-C Liou stated that,

"The 'removing five objects per year can stabilize the LEO environment conclusion' is somewhat notional. It is intended to serve as a benchmark for ADR planning."

(J.C. Liou, Presentation at the 2nd European Workshop on Active Debris Removal, CNES HQ, France, slide 19, June 2012) 
The way we utilise near-Earth space, and the way the space environment behaves in the longterm future will directly affect the number of debris objects required to be removed. New space technologies, increasing numbers of space-faring nations, developing policies and political motivations will affect launch and mitigation activity, and thus the size of the LEO population, potentially threatening the sustainability of outer space activities. These future values remain unknown. Results from White and Lewis (2014) have shown that modifying values for launch, explosion, mitigation and solar activity, as well as looking at objects down to sizes of five $\mathrm{cm}$ can significantly effect on the sustainability of space activities, even whilst utilising ADR of five and ten removals per year.

Fig. 1 highlights the difference between debris population of substantial and non-mitigation scenarios from the University of Southampton's Debris Analysis and Monitoring Architecture for the Geosynchronous Environment (DAMAGE) tool. The grey line indicates the 100 Monte Carlo (MC) average LEO population (objects $\geq 10 \mathrm{~cm}$ ) with the same study set up as (IADC, 2013). Whereas, the black line shows the average debris population results of the non-mitigation scenario. The future debris population, without ADR, is then likely to be between these two curves. Given that, over the last 30 years strides in compliance with mitigation have been increasing, the most realistic population is likely to be closer to the AI27.1 line.

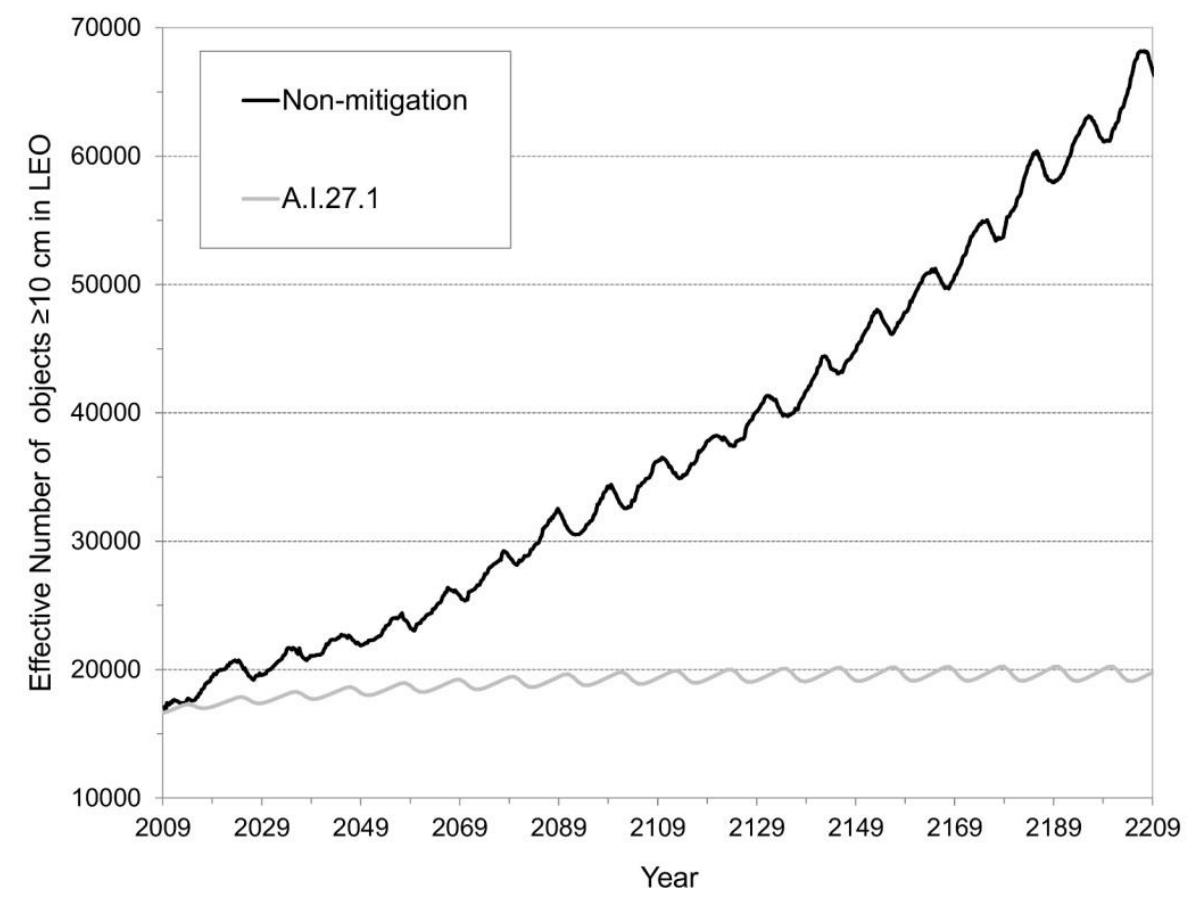

Fig. 1. A comparison of DAMAGE results between strong (grey) and no (black) mitigation of LEO objects $\geq 10 \mathrm{~cm}$. 
It may be difficult then, to answer the question: how many objects should be removed via ADR? Having a well-thought-out long-term strategy to establish this will ensure the most effective use of ADR and best outcome for the environment. Further, it may help to drive other ADR challenges, including which objects to remove, the choice of ADR technology and allow for a greater understanding of financial costs.

This work outlines a long-term strategy to help determine how many debris objects should be removed to control the size of the LEO debris population. The strategy implemented was to adapt and adjust the number of removals per year (or ADR rate) performed by ADR in response to the evolution of the debris population. The strategy therefore, does not directly attempt to reduce future uncertainty but, by being flexible, it adapts to events, information and motivations that may have or have already affected the debris population. This type of approach is known as an adaptive strategy (AS).

Holling (1978) and Walters (1986) primarily developed this adaptive response in the 1970's for environmental and renewable resource uncertainty, commonly referred to as adaptive management. Whilst adaptive management has various interpretations, a simple definition by Holling (1978) is the systematic process for continually improving management policies and practices by learning from the outcomes of programs. An AS will vary, depending upon the problem, level of uncertainty and predictive capability but the same six step framework of activities is typically used (Fig. 2).

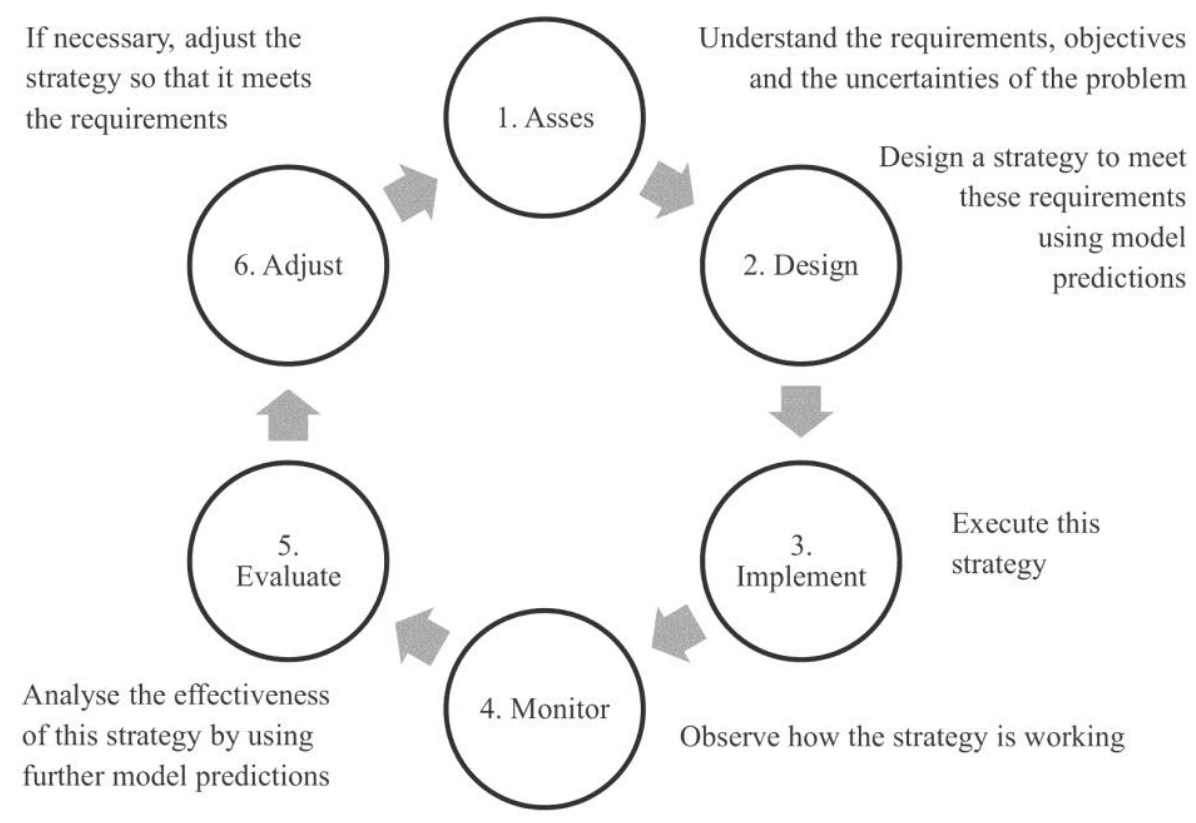

Fig. 2. The framework for an adaptive strategy (Nyberg, 1999).

In this work, the framework in Fig. 2 was used as a starting point to develop a long-term AS to determine the number of debris objects to be removed in the future. Within the AS, a new statistical evolutionary model was used, entitled the Computational Adaptive Strategy to Control Accurately the 
Debris Environment (CASCADE). CASCADE, although being a generalised model, is capable of predicting statistically the long-term LEO debris population, whilst incorporating the effects of ADR. As it is impractical to test the AS on the space environment, instead the real space environment was represented by the University of Southampton's evolutionary debris model, the Debris Analysis and Monitoring Architecture for the Geosynchronous Environment (DAMAGE), which performed multiple projections of the $\geq 10 \mathrm{~cm}$ LEO debris population over 200 years. DAMAGE is a threedimensional computational model capable of predicting the evolution of the full LEO to GEO space debris environment. More information, and recent studies of DAMAGE include the effects of thermosphere contractions on ADR (Lewis et al., 2011) and investigating synergies between debris ADR and mitigation (Lewis et al., 2012).

Every five years of each DAMAGE projection, CASCADE predicted the long-term debris population, using the current DAMAGE debris population as an initial condition. CASCADE made several predictions with differing ADR rates, until an appropriate ADR was found that stopped the growth of the $\geq 10 \mathrm{~cm}$ LEO population in the current projection. This removal rate was then implemented with the DAMAGE projection for five years, and then recalculated.

The results of this AS were compared with three different scenarios: a scenario with no ADR and two scenarios whereby three or five objects were removed per year. Each scenario used a MC simulation technique that comprised of 100 future projections.

\section{The Adaptive Strategy}

The framework in Fig. 2 was modified to create the AS used within DAMAGE. In Fig. 2, step (1)'s purpose is to analyse and determine the high-level requirements for ADR activities. In this study, the high-level requirement for ADR was to prevent the growth of the $\geq 10 \mathrm{~cm}$ LEO population over 200 years, and this did not change throughout the DAMAGE projection. This then makes it consistent with previous ADR studies, such as Lewis et al. (2009a) and Liou et al. (2010), allowing an easier comparison between studies to be made. As this requirement did not change, it was unnecessary to replicate steps (1) through (3) in Fig. 2 more than once, as these steps are used, more generally, to assess and design strategies based on new requirements. Thus, the AS can be simplified by only repeating the steps (4)-(6).

On 1 May 2009, each DAMAGE projection commenced. On 1 Jan 2020, ADR activities started. An assessment of the debris population was made by CASCADE and the requirement for ADR activities was selected to be to prevent the growth of the $\geq 10 \mathrm{~cm}$ LEO debris population over 200 years (step (1)).

In order to design a strategy to meet this requirement, step (2), DAMAGE passed the number of $\geq 10 \mathrm{~cm}$ LEO objects, $N_{D}(t)$, to CASCADE, which then predicted the long-term LEO population, $N(t, \dot{\mathrm{R}})$, where $\dot{\mathrm{R}}$ is equal to the yearly $\mathrm{ADR}$ rate. 
The first CASCADE projection was without $\mathrm{ADR}, N(t, \dot{\mathrm{R}}=0)$. At the end of the projection on 1 May 2209, the CASCADE population, $N\left(t_{e}, \dot{\mathrm{R}}=0\right)$, was compared with the initial population of the DAMAGE projection, $N_{D}\left(t_{0}\right)$. Such that

$N_{p}=N\left(t_{e} \dot{\mathrm{R}}\right)-N_{D}\left(t_{0}\right)$

where $t_{e}$ the projection end time (1 May 2209). To find the value of $\dot{\mathrm{R}}$ where $N_{p}=0$, which reflects the requirement of no growth, CASCADE was run multiple times with different values of $\dot{\mathrm{R}}$, using a bisection method. The initial values of $\dot{R}$ were 0 and 20 removals per year. The bisection method was halted when a $\dot{R}$ value was found to the nearest integer. This removal rate, $\dot{R}$, was then implemented for five years in the DAMAGE projection (step (3)). Five years was selected to allow time to establish the effect of ADR, whilst still being able to react to events that occur within a reasonable period.

Step (4) calls for continuous monitoring of the debris population and the effectiveness of ADR. However, in this study the DAMAGE LEO population was only monitored at each for five-year interval when an evaluation of the environment was made (step 5). The evaluation used the same process outlined above, and adjusted the ADR rate if necessary (step (6)) until the DAMAGE projection was completed on 1 May 2209.

\section{The CASCADE Model}

CASCADE is a statistical model designed to predict the long-term evolution of the $\geq 10 \mathrm{~cm}$ LEO debris population whilst incorporating the effect of ADR. There have been a number of previous statistical models, which have used a similar approach; these include mathematical models to describe the state of the future environment, such as Kessler and Cour-Palais (1978), Reynolds et al. (1983). To more recent models such as Talent's (1992) particle-in-a-box (PIB) model, NASA's CHAIN model (later CHAINEE) (Rex and Eichler, 1993), Nazarenko's (1993) model, the stochastic analog tool (Rossi et al., 1995), the stochastic impressionistic low Earth model (Ananthasayanama et al., 2006) and the University of Southampton's Fast Debris Evolution (FADE) model (Lewis et al., 2009b).

The main advantage of using a statistical approach over complex semi-analytical codes is the speed at which long-term predictions can be made. This is important, as hundreds of future long-term projections were required investigate a wide range of ADR rates, for every DAMAGE projection. The main disadvantage however, is that statistical models are limited to analysing only a small set of states about the debris environment, such as total number of objects, collisions, spatial density. In the context of this work it was not an issue as only the total number of objects in LEO was required from CASCADE. 
The rate of LEO population change over time was described by a non-linear, first-order, ordinary differential equation to determine the future debris population. The number of objects in orbit at time $t, N(t, \dot{R})$, was split into three debris types: intact objects, $N i(t)$, explosion fragments, $N e(t)$, and collision fragments, $N c(t)$. Where, $\dot{R}$, refers to the rate of change of the yearly number of removals via ADR. The rate of change of $N(t, \dot{\mathrm{R}})$ with respect to time was

$\dot{N}(t, \dot{\mathrm{R}})=\dot{N} i(t)+\dot{N} e(t)+\dot{N} c(t)$.

Given $N i, N e$ and $N c$ at the start time, $t_{0}$, the differential equation for category was solved for future time steps numerically using Euler's method. For example, the number of intact objects was calculated by

$N i(t+\Delta t)=N i(t)+\dot{N} i \Delta t$

For a small time step value ( $\Delta t=1$ day) the error using Euler's method was considered to be acceptable at $0.05 \%$ per year.

The LEO region was discretised into nine altitude bands each $200 \mathrm{~km}$ in altitude between 200 and 2,000 km. CASCADE is only a generalisation of LEO and therefore makes only an approximate prediction of the number of objects in LEO, therefore some assumptions about object properties and orbits were assumed. As a result, individual objects resided in only one altitude band and can only move to lower altitude bands by decaying. This implies that the orbits of all objects are circular or near circular. Thus, an object's semi-major axis was used to determine which altitude band was used.

Objects below $200 \mathrm{~km}$ in semi-major axis were not included within the model, as the minimum altitude modelled in DAMAGE is $200 \mathrm{~km}$. It was assumed that these objects would decay within a small time period. As such these objects would not globally affect the size or evolution of the LEO population.

Eq. (2) was separated into nine separate summations of each altitude band

$\dot{N}(\dot{R})=\sum_{h=1}^{9} \dot{N} i_{h}+\sum_{h=1}^{9} \dot{N} e_{h}+\sum_{h=1}^{9} \dot{N} c_{h}$

For clarity, the notation indicating time dependence $(t)$ has been dropped. The subscript $h$ is equal the altitude band number, which ranged from $h=1$ referring to the lowest altitude band (200-400 km), and $h=9$ denoting the highest altitude band (1,800-2,000 km).

The rate of change of intact objects, $\dot{N} i$, was equal to 
$\dot{N} i=\sum_{h=1}^{9}\left(\dot{L}_{h} n_{L}+\dot{D} i_{h+1} N i_{h+1}-\dot{D} i_{h} N i_{h}-\dot{E}_{h}-\dot{C}_{h}-\dot{P}_{h} \dot{L}_{h} n_{L}-\dot{R}_{h}\right)$

where

- $\dot{L}_{h} n_{L}$ is the rate of intact objects launched. This was the product of the launch rate, $\dot{L}_{h}$, and the number of objects per launch. Whilst the real value maybe slightly higher, for simplicity, it was assumed, in this case, that no mission-related debris were included and that that one satellite and one rocket body was released per launch, such that $n_{L}=2$,

- $\dot{D} i_{h+1} N i_{h+1}$ is the rate of intact objects that have decayed from the higher altitude band, $\dot{D} i_{h+1}$ is the drag rate of the higher altitude band,

- $\dot{D}_{i_{h}} N_{i_{h}}$ is the number of intact objects that have decayed out of the altitude band,

- $\dot{E}_{h}$ is the intact object explosion rate,

- $\dot{C}_{h}$ is the intact object collision rate,

- $\dot{P}_{h} \dot{L}_{h} n_{L}$ is the rate intact objects complying with PMD,

- $\dot{R}_{h}$ is the rate of intact objects removed by ADR.

The rate of change of explosion fragments was

$\dot{N} e=\sum_{h=1}^{9}\left(\dot{E}_{h} n_{e}+\dot{D} e_{h+1} N e_{h+1}-\dot{D} e_{h} N e_{h}\right)$

where $\dot{E}_{h} n_{e}$ is equal to the rate of number of explosion fragments generated. This was the product of the explosion rate, $\dot{E}_{h}$, and the average number of explosion fragments generated per explosion, $n_{e}$. The number of fragments generated for each explosion was dependent on the mass of the object and the energy of the explosion. To reproduce an average number of explosion fragments the NASA standard breakup model was utilised (Johnson et al., 2001)

$n_{e}=6 F L_{c}{ }^{-1.6}$,

where $L_{c}$ is equal to the minimum characteristic length (average of the three dimensions) of the fragments $(0.1 \mathrm{~m})$, and $F$ is a scaling factor, that in this case was kept at 1 . The number of generated fragments $\geq 10 \mathrm{~cm}$ that resulted then was 239 . It was assumed that generated explosion fragments 
would remain in the same altitude band initially and have a negligible velocity change with respect to the "parent" object.

Finally, the rate change of collision fragments was

$$
\dot{N} c=\sum_{h=1}^{9}\left(\dot{C}_{h} n_{c}+\dot{D} c_{h+1} N c_{h+1}-\dot{D} c_{h} N c_{h}\right) \text {, }
$$

where $\dot{C}_{h} n_{c}$ is equal to the rate of number of collision fragments generated. This was the product of the intact object collision rate, $\dot{C}_{h}$, and the average number of collision fragments generated per collision, $n_{c}$. The number of fragments generated from a collision is dependent upon the mass of the objects and their relative velocity. To reproduce an estimate of this number, the NASA standard break-up model was used (Johnson et al., 2001), such that

$n_{c}=0.1\left(2 m_{\text {avg }}\right)^{0.75} L_{c}{ }^{-1.71}$,

where, $m_{\text {avg }}$ is equal to the average mass of an intact rocket body or satellite $(644 \mathrm{~kg})$, the number of collision fragments for the application of the model was 1,103.

The collision rate for each band, $\dot{C}_{h}$, was calculated using an empirical equation

$\dot{\mathrm{C}}_{h}=k_{1}+k_{2} I_{h}+\mathrm{k}_{3} I_{h}^{2}-\mathrm{k}_{4} \dot{R}_{h}$,

this was a derivative of FADE (Lewis et al., 2009b) and Talent (1992) approach. Where $k_{1}$ through $k_{3}$ were coefficients of the collision rate and $I_{h}$ accounted for all the possible interactions between objects (for example intact-intact, intact-collision fragment etc.) and was

$I_{h}=N i_{h}^{2}+N e_{h}^{2}+N c_{h}^{2}+N i_{h}\left(N e_{h}+N c_{h}\right)+N e_{h} N c_{h}$.

The coefficients $k_{1}$ through $k_{3}$ were required, such that the collision rate can be tuned with DAMAGE to give the correct rate within CASCADE. The collision coefficient $k_{4}$ determined the reduction in collision probability that occurred when an object was removed from the environment using ADR.

To determine the collision coefficient values $\left(k_{1}-k_{4}\right)$, eight studies were performed using DAMAGE (50 MC each), between 2009 and 2209, with ADR rates of 0, 1, 2, 3, 4, 5, 8, 10 objects per 
year. Included within these studies were widespread mitigation compliance (90\% compliance with a 25-year PMD rule, no explosions) and an eight-year repeated launch traffic cycle. The ADR target selection criterion used by DAMAGE, $T_{i}(t)$, based on minimising the risks of a collision (Liou and Johnson, 2009), was

$T_{i}(t)=P_{i}(t) \times m_{i}$,

where $m_{i}$ is the mass of an intact object $i$ and $P_{i}(t)$ is the total collision probability of object $i$ at a specific time $t$. Objects with the highest mass-collision probability were removed first.

Using the outputs of these DAMAGE studies, the coefficients were $k_{1}=0, k_{2}=3.8 \times 10^{-7}, k_{3}=$ $1.2 \times 10^{-1}$ and $k_{4}$ as a function of $\dot{R}$, was found to be

$k_{4}=\left\{\begin{array}{ll}0.02-0.001 \dot{R} & \dot{R}<5 \\ 0.014 & \dot{R} \geq 5\end{array}\right.$.

Fig. 2 shows the LEO populations for a variety of these ADR rates, for both CASCADE and DAMAGE. The coefficient of determination $\left(R^{2}\right)$ between the DAMAGE and CASCADE populations were between 0.96-0.99.
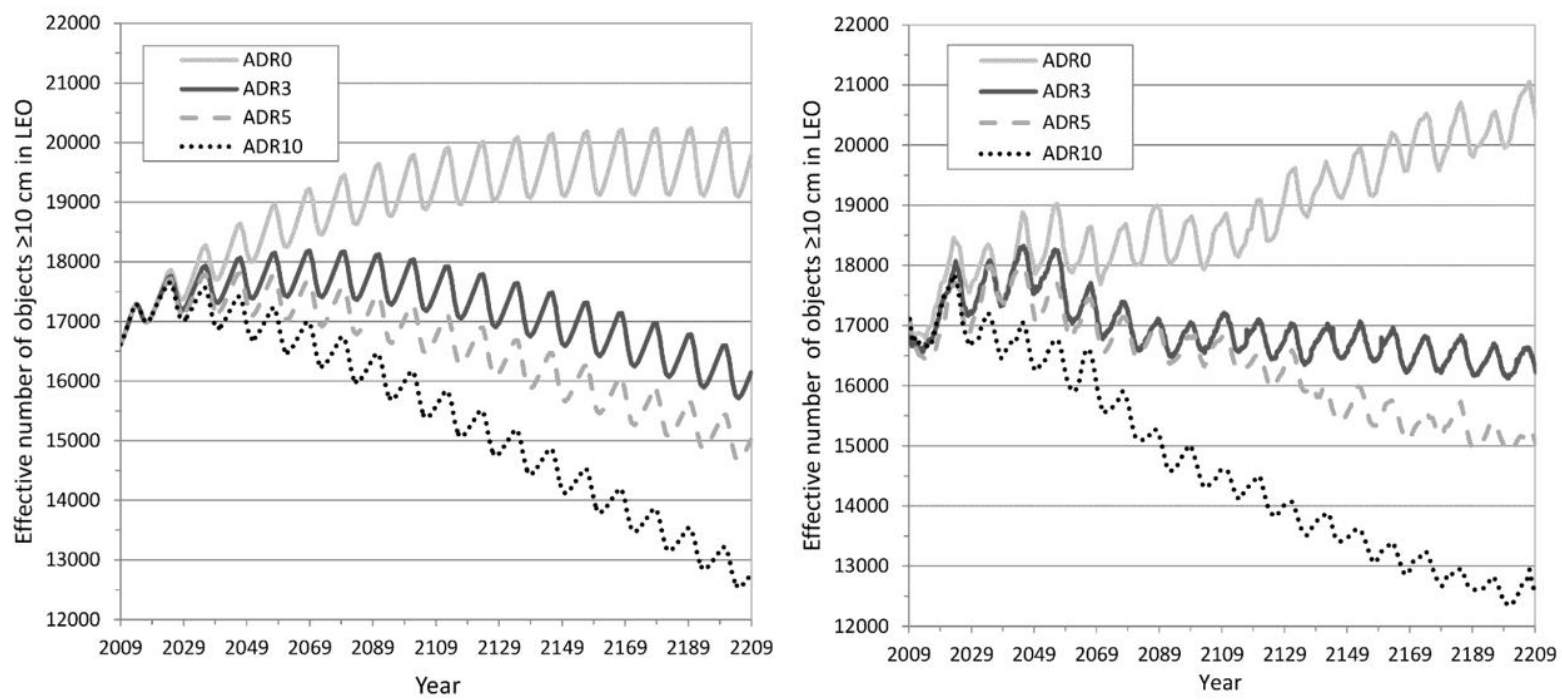

Fig. 3. A Comparison of CASCADE (left) and DAMAGE (right) LEO debris populations for no, three, five and 10 removals per year.

The drag rates, representing the proportion of objects that decayed from a particular altitude band per year, for intact $\left(\dot{D} i_{h}\right)$, explosion $\left(\dot{D} e_{h}\right)$ and collision $\left(\dot{D} c_{h}\right)$ were calculated from the average size of each object category, and an atmospheric density value calculated from the Naval Research 
Laboratory Spectrometer and Incoherent Scatter Radar (NRLMSIS-00) model in the middle of each altitude band. These values are shown in Table 1 .

Table 1. Drag rates, implemented in CASCADE, for intact, explosion and collision objects.

\begin{tabular}{llll}
\hline $\begin{array}{l}\text { Altitude } \\
\text { band [km] }\end{array}$ & $\begin{array}{l}\text { Intact object drag } \\
\text { rate [\#/year] }\end{array}$ & $\begin{array}{l}\text { Explosion object } \\
\text { drag rate [\#/year] }\end{array}$ & $\begin{array}{l}\text { Collision object } \\
\text { drag rate [\#/year] }\end{array}$ \\
\hline $200-400$ & 0.55 & 0.55 & 0.55 \\
$400-600$ & 0.0045 & 0.0055 & 0.017 \\
$600-800$ & 0.0015 & 0.0021 & 0.0052 \\
$800-1,000$ & 0.0014 & 0.0016 & 0.0054 \\
$1,000-1,200$ & 0.0014 & 0.00079 & 0.0045 \\
$1,200-1,400$ & 0.00092 & 0.00038 & 0.0032 \\
$1,400-1,600$ & 0.00073 & 0.00056 & 0.0020 \\
$1,600-1,800$ & 0.00059 & 0.00043 & 0.0021 \\
$1,800-2,000$ & 0.00053 & 0.00021 & 0.0019 \\
\hline
\end{tabular}

A scaling factor $S(t)$ was used to replicate the effect of solar activity on the drag rate of debris. $S(t)$ was derived from the arithmetic average of the last four complete solar cycles F10.7 cm values. These data were acquired from the National Oceanic and Atmospheric Administration National Geophysical Data Centre (2013). The yearly values of $S(t)$ for one complete solar cycle are shown in Table 2. This scaling factor was multiplied by the values in Table 1 to give the correct drag rate for each year. The values in Table 2 repeat throughout the projection period.

Table 2. Yearly values of $S(t)$ over one complete solar cycle.

\begin{tabular}{clllllllllll}
\hline & & \multicolumn{1}{c}{ Years After 2009 } \\
Parameter & $\mathbf{0}$ & $\mathbf{1}$ & $\mathbf{2}$ & $\mathbf{3}$ & $\mathbf{4}$ & $\mathbf{5}$ & $\mathbf{6}$ & $\mathbf{7}$ & $\mathbf{8}$ & $\mathbf{9}$ & $\mathbf{1 0}$ \\
\hline$S(t)$ & 1.00 & 1.00 & 1.07 & 1.42 & 1.69 & 1.78 & 1.70 & 1.52 & 1.30 & 1.11 & 1.03 \\
\hline
\end{tabular}

The PMD compliance rate, $\dot{P}$, was expressed as the proportion of objects per year that complied with PMD guidelines outlined in the IADC Space Debris Mitigation Guidelines (IADC, 2007). It was assumed that moving objects into altitudes between 200 and $600 \mathrm{~km}$ made sure these intact objects decayed out of the projection within 25 years.

Fig. 4 illustrates a comparison between the average population output of a 50 MC DAMAGE study and the output of a CASCADE prediction, both without ADR. This study was implemented with $90 \%$ compliance with a 25-year PMD rule, no explosions and an eight-year repeated launch traffic cycle. 


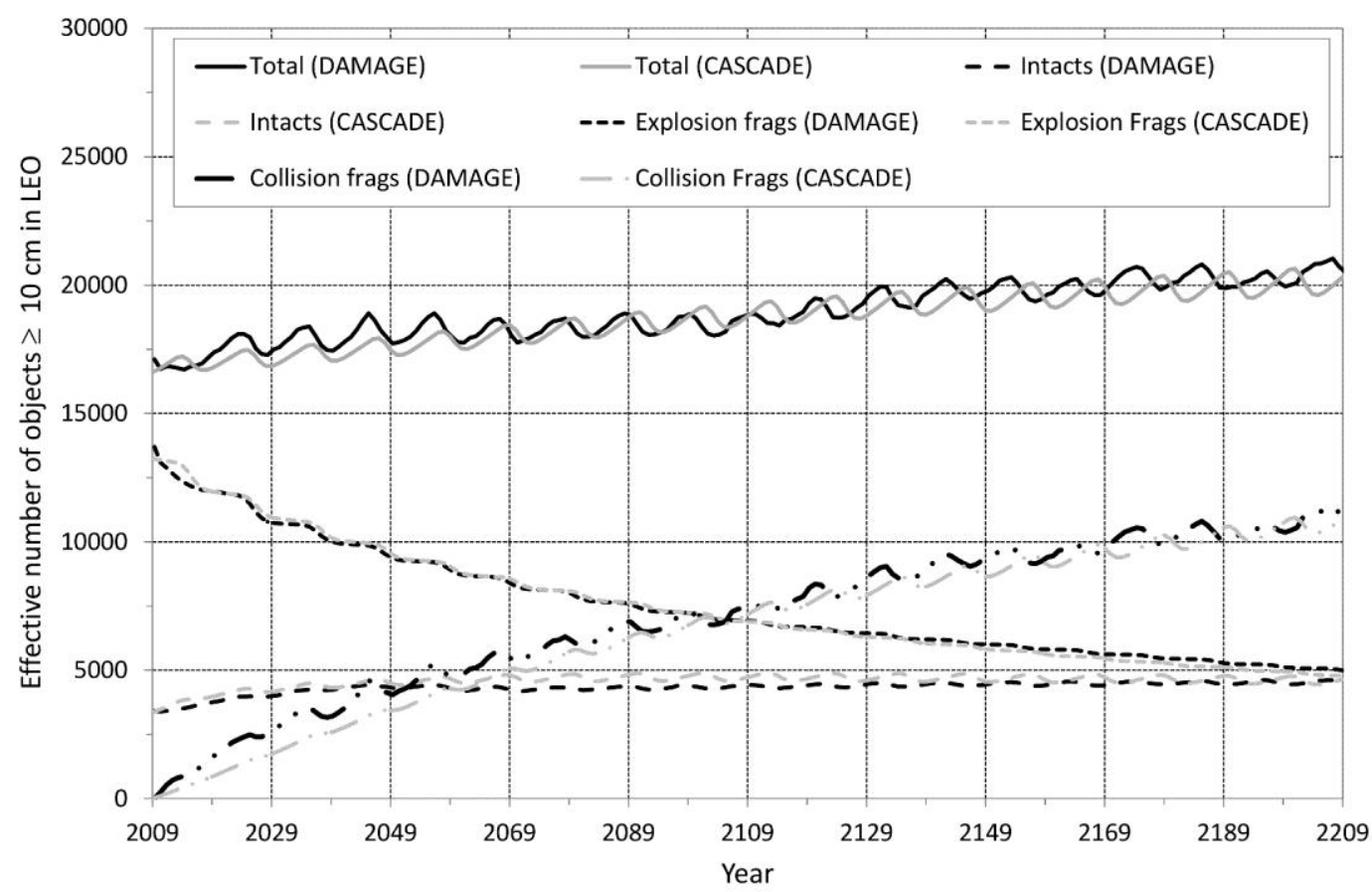

Fig. 4. A comparison of the number of objects between DAMAGE and CASCADE, separated by object type.

\section{Description of Study}

Along with the adaptive strategy (AS), three other scenarios were investigated: the removal of three objects per year (ADR3), five objects per year (ADR5) and a baseline scenario with no ADR (ADR0). These scenarios, henceforth, are referred to by their acronyms. ADR0 acted as a baseline scenario whilst removing three and five objects per year replicated previous ADR modelling studies. A summary of the key study parameters and their implementation in DAMAGE (Table 3) and CASCADE (Table 4) are shown.

The simulation set up was the same as (IADC, 2013) and other previous ADR modelling studies (Klinkrad and Johnson, 2010; Liou et al., 2010; Lewis et al., 2012). This way the effectiveness of the adaptive strategy can be easily seen and related to previous works. 
Table 3. Key study parameters and how they were implemented in DAMAGE.

\begin{tabular}{|c|c|}
\hline Parameter & DAMAGE implementation \\
\hline Projection period & 1 May 2009 - 1 May 2209 \\
\hline Initial population & $\begin{array}{l}\text { Meteoroid and Space Debris Terrestrial Environment Reference (MASTER) } \\
\text { population } \geq 10 \mathrm{~cm} \text { residing in or passing through the LEO regime on } 1 \text { May } \\
2009\end{array}$ \\
\hline Object sources & Explosion and collision fragments, operational and defunct satellites and rocket \\
\hline modelled & bodies \\
\hline Solar activity & $\begin{array}{l}\text { An F10.7 cm solar cycle, derived from the arithmetic average of the last four } \\
\text { complete solar cycles, was used and repeated every } 11 \text { years }\end{array}$ \\
\hline Launch traffic & $\begin{array}{l}\text { Repeated 8-year (2001-2009) launch traffic, acquired from ESA's Database } \\
\text { and Information System Characterising Objects in Space (Flohrer et al., 2013) }\end{array}$ \\
\hline Explosions & No explosions \\
\hline PMD & $\begin{array}{l}\text { Spacecraft and rocket bodies were moved immediately to orbits that decay } \\
\text { within } 25 \text { years ( } 1 \text {-year tolerance) or re-orbited above LEO and taken out of the } \\
\text { projection with a } 90 \% \text { success rate }\end{array}$ \\
\hline ADR start time & 1 Jan 2020 \\
\hline $\begin{array}{l}\text { ADR selection } \\
\text { criterion }\end{array}$ & $\begin{array}{l}\text { Eq. (1) was used. Objects were also intact, removed immediately from the } \\
\text { projection, have an orbital eccentricity }<0.5 \text { and a perigee altitude }<1,400 \mathrm{~km}\end{array}$ \\
\hline Time step & 5 days \\
\hline $\begin{array}{l}\text { Satellite } \\
\text { properties }\end{array}$ & $\begin{array}{l}\text { The operational lifetime was set to eight years and no collision avoidance } \\
\text { manoeuvres occurred }\end{array}$ \\
\hline
\end{tabular}

Table 4. Key study parameters and how they were implemented in CASCADE. Where the parameters in Table 3 have not been repeated, they have been implemented the same way as DAMAGE.

\begin{tabular}{ll}
\hline Parameter & CASCADE implementation \\
\hline Projection period & 1 Jan 2020 - 1 May 2209 \\
Initial population & DAMAGE population $\geq 10 \mathrm{~cm}$ at $1 \mathrm{Jan} 2020$ \\
PMD & $\begin{array}{l}\text { Intact objects with altitudes greater than } 800 \mathrm{~km} \text { were moved immediately } \\
\text { between altitudes of } 400-800 \mathrm{~km} \text { with a } 90 \% \text { success rate }(\dot{\mathrm{P}}=0.9)\end{array}$ \\
ADR selection & Any object in the altitude band with the highest collision probability was \\
criterion & removed. Object must be intact and removed immediately from the projection \\
Time step & 1 day \\
\hline
\end{tabular}

The minimum object size was $10 \mathrm{~cm}$; this represented the historical detection threshold limit for the Space Surveillance Network sensors in LEO and accounts for approximately $97 \%$ of the total mass in LEO. 
One hundred MC projections were carried out for each scenario to obtain the arithmetic average and standard deviation of the $\geq 10 \mathrm{~cm}$ LEO population. In addition, the proportion of projections demonstrating a decrease in population after 200 years as well as the cumulative number of collisions, both catastrophic and non-catastrophic, were recorded and analysed for each projection.

An effective reduction factor (ERF) (Liou and Johnson, 2009), was calculated to quantify the effect of ADR. The ERF is defined as the number of debris objects reduced in the environment using ADR over a period, $t$, divided by the number of objects removed by ADR. Such that

$\operatorname{ERF}(t)=\frac{N_{A}(t)-N_{B}(t)}{C N_{R}(t)}$

where $N_{A}(t)$ is the number of objects $\geq 10 \mathrm{~cm}$ in the ADR0 scenario, $N_{B}(t)$ is the number of objects $\geq 10 \mathrm{~cm}$ for an ADR scenario (ADR3, ADR5 or AS) and $C N_{R}(t)$ is the cumulative number of objects removed for the ADR scenario by time $t$. The ERF was measured at the end of the projection $\left(t_{e}\right)$ on 1 May 2209.

\section{Results}

The $100 \mathrm{MC}$ arithmetic average of the $\geq 10 \mathrm{~cm}$ LEO debris population for each scenario is presented in Fig. 5. A summary of results for each scenario, including the standard deviations of the populations and the cumulative number of collisions is shown in Table 5.

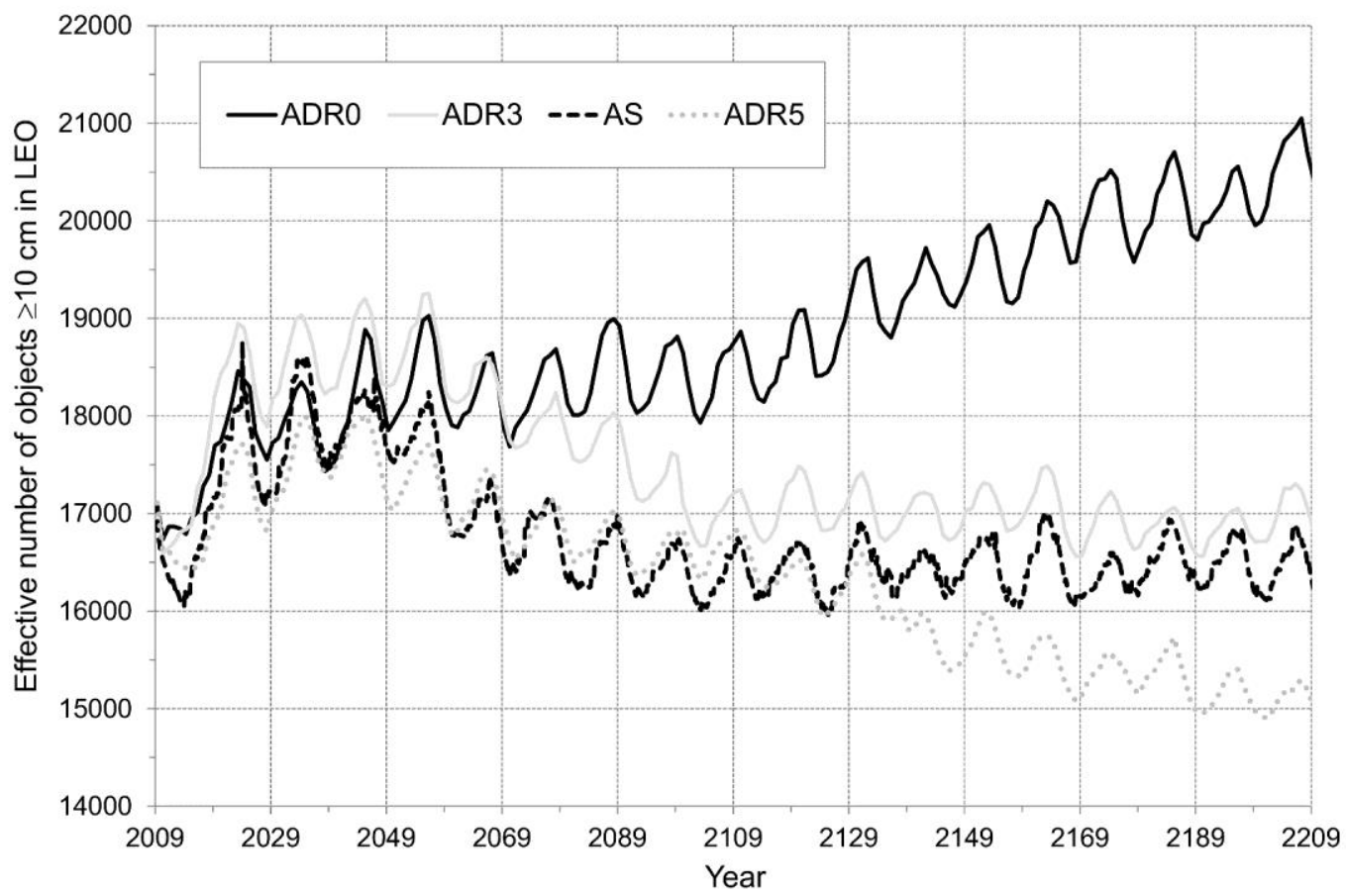

Fig. 5. The 100 MC arithmetic average of objects $\geq 10 \mathrm{~cm}$ for each scenario. 
The $R^{2}$ values were 0.98 for intact objects, 0.98 for explosion fragments, 0.97 for collision fragments and 0.97 for the total number of objects.

On average, each scenario with ADR was able to stop the growth of the LEO population whilst the population in ADR0 grew by $20 \%$. The removal of five objects per year showed the biggest decrease, with a $12 \%$ population reduction compared with the initial population on average. This was followed by the AS scenario with a 5\% decrease and the ADR3 scenario with a $2 \%$ decrease. However, $42 \%$ of ADR3 projections' populations still saw a growth. Even when removing five objects per year, $26 \%$ of projections' populations still grew in size. The AS performed best, with only $12 \%$ of projections' populations being above the initial population. The cumulative and catastrophic number of collisions follows the same as the total number of objects.

Table 5. Summary of arithmetic average results for each scenario. Values in brackets show one standard deviation.

\begin{tabular}{lllll}
\hline Scenario & $\begin{array}{l}\text { Average LEO } \\
\text { population } \mathbf{1 0} \mathbf{~ c m} \\
\text { after 200 years }\end{array}$ & $\begin{array}{l}\text { Percentage of } \\
\text { projections below } \\
\mathbf{2 0 0 9} \text { population }\end{array}$ & $\begin{array}{l}\text { Average cumulative } \\
\text { number of collisions }\end{array}$ & $\begin{array}{l}\text { Average number } \\
\text { of catastrophic } \\
\text { collisions }\end{array}$ \\
\hline ADR0 & $20,450(3,660)$ & $16 \%$ & $63.4(14.8)$ & $37.8(8.6)$ \\
ADR3 & $16,830(3,630)$ & $58 \%$ & $51.9(12.3)$ & $33.7(8.3)$ \\
ADR5 & $15,040(3,390)$ & $74 \%$ & $41.8(9.1)$ & $27.3(7.4)$ \\
AS & $16,240(1,720)$ & $88 \%$ & $43.2(5.9)$ & $29.4(3.6)$ \\
\hline
\end{tabular}

The biggest difference between the AS and other scenarios was the variance and range between individual projections. The one standard deviation of the populations for the ADR3 and ADR5 scenarios were over double that of the AS scenario. To highlight this, Fig. 6 shows a histogram all the AS and ADR5 projections' populations after 200 years, in bins sizes of 500 objects. Each bin shows the number of MC projections that had a particular debris population at the 2209 epoch. This histogram therefore illustrates the distribution of debris populations between the two scenarios. The shaded portion of Fig. 6 indicates the region where the population had grown in comparison to the initial population.

The ADR5 scenario had over double the range in population after 200 years compared with the AS scenario. The highest ADR5 projection population at the 2209 epoch was 24,770 objects and the lowest was 10,908 objects. Whereas, the highest AS projection population was 13,701 objects and lowest was 18,461 objects. 


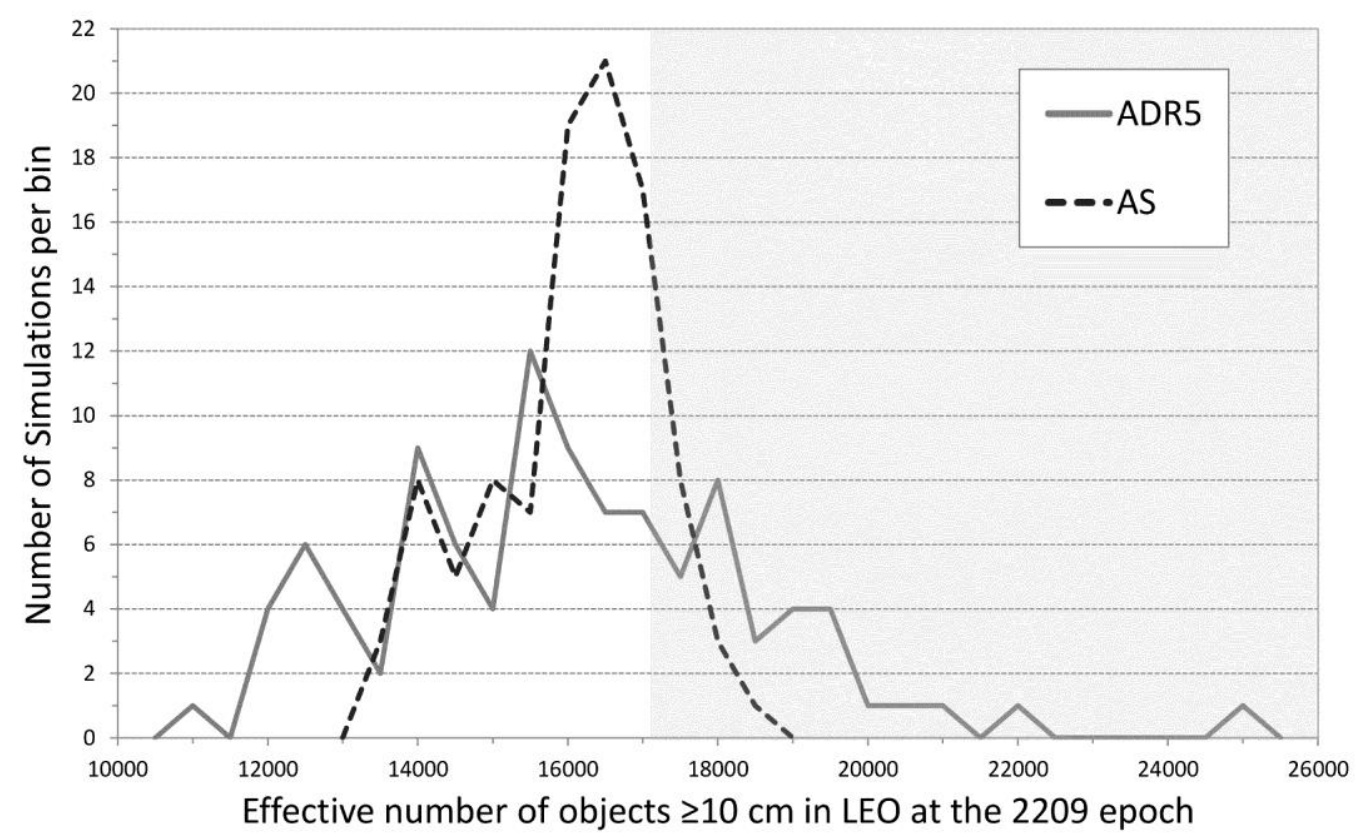

Fig. 6. Histogram of all the LEO debris populations for the AS and ADR5 scenarios at the 2209 epoch. Bin sized equal to 500 objects. The grey shaded area represents all projections that have grown in size after 200 years.

A summary of ADR statistics for each scenario is shown in Table 6. The number of removals required to prevent a collision from occurring in the AS scenario was in the order of two-thirds that of the other scenarios.

Table 6. A Summary of ADR statistics, value in brackets shows a one standard deviation.

\begin{tabular}{llll}
\hline Scenario & $\begin{array}{l}\text { Average removal } \\
\text { rate [\#/year] }\end{array}$ & $\begin{array}{l}\text { Average } \\
\text { ERF }\end{array}$ & $\begin{array}{l}\text { Average number of removals } \\
\text { required to prevent a collision }\end{array}$ \\
\hline ADR3 & 3 & 6.4 & 44 \\
ADR5 & 5 & 5.7 & 49 \\
AS & $3.1(1.2)$ & 7.2 & 29 \\
\hline
\end{tabular}

Fig. 7 illustrates a histogram of the average removal rates, over the time ADR was implemented, for each AS projection. Only $11 \%$ of the AS projections required a removal of greater than five objects per year. In fact, over half of the projections required fewer than three removals per year. 


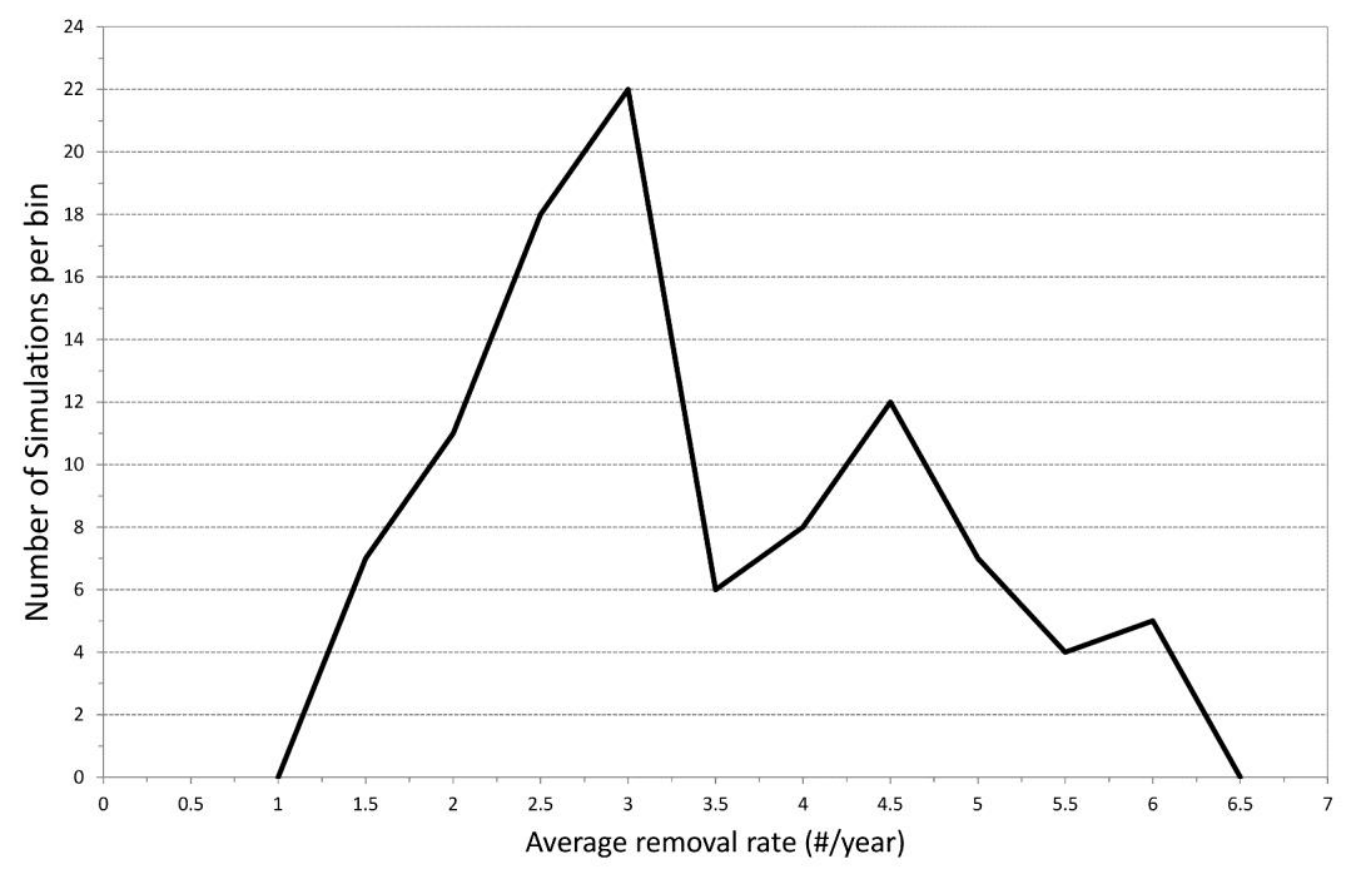

Fig. 7. Histogram of the average yearly ADR rate for all AS projections. Bin size equal to 0.5.

\section{Discussion}

It is clear that the AS is preferable to a fixed removal rate. The AS had a $12 \%$ higher probability of achieving zero growth compared with five removals per year. To accomplish this, the average number of required removals was fewer, 3.1 per year, compared with the typically quoted five objects per year. This, therefore, shows such a strategy is both more efficient (requiring only $61 \%$ of the number of removals) and more effective (26\% higher probability of being below the initial population in 2009) compared with removing five objects a year throughout the projection period.

The AS has a greater degree of control over the population; almost all projections were within a narrow range of the requirement to prevent population growth. This is important, as in some projections removing five objects per year without adapting is not enough to prevent growth, and in others projections no removals were required to prevent growth. In reality, if ADR is implemented routinely and the debris population continues to grow the number of removals will be increased. Conversely, if the population is reducing, the number of removals may too be reduced. The AS then represents a more realistic implementation of ADR within modelling studies. As a caveat, the focus here has been primarily to demonstrate the feasibility of different approach to utilising ADR, and not provide exact future ADR rates to ensure that the long-term population will not grow.

None of the scenarios demonstrated in this paper guaranteed that the long-term debris population would not grow. This is due to the requirement selected, as the measure of success was to assess the number of debris objects at the end of each projection. It is possible to reform this requirement to address this issue. For example modifying the requirement to stop the growth of the average number 
of objects over a set number of years or allowing a controlled growth of the population, not exceeding a set number of collisions, or even to reduce the population would be equally valid but would require different sets of strategies.

The use of an AS is not limited to adapting the number of removals required per year. It may incorporate other parameters that can help control the LEO space debris population, such as launch rates and the implementation of mitigation measures like PMD. Incorporating additional parameters gives the additional benefit of flexibility, as well as potentially a greater degree of control over the environment.

Finally, the DAMAGE projections in this paper were based on a set of assumptions that constrained the launch, explosion, solar and mitigation activity. The projections were based around the same study set up as (IADC, 2013), which was considered optimistic. Particularly, as launch rates may be increasing and mitigation is not yet at the level of compliance projected in this work. As a result, the AS has only been established and quantified for a narrow range of possible outcomes. This was done to show the effectiveness of such a strategy against previous studies using ADR with fixed removal rates. Therefore, results in this paper do not represent the mode likely future, and as such the removal rates shown are intended only to serve as a preliminary guide with respect to the AS method.

Further work will be to incorporate a degree of randomness in these aforementioned parameters and further investigate the effectiveness and advantages of the AS. This degree of randomness implemented to future parameters has already been established in work by White and Lewis (2014).

\section{Conclusion}

Using the University of Southampton's DAMAGE and CASCADE models, a long-term adaptive strategy for ADR rates to help control the LEO space debris population has been developed. By monitoring the environment and adjusting, if necessary, ADR rates, an AS can provide a method that is more effective at achieving a sustainable long-term space debris population compared with the maladaptive alternative. Often this approach is more efficient, requiring fewer removals.

Due to the positive results, it is recommended that following studies involving ADR begin to include some sort of a monitoring and adjusting approach to provide a realistic representation of how ADR may be utilised in the future. We advocate continued research by nations, committees, organisations and others in order to determine the high-level requirements of ADR and to address the political, economic and technological challenges that go with it. 


\section{Acknowledgements}

Financial support for this work was provided by the University of Southampton's Faculty of Engineering and the Environment. The authors express thanks to Holger Krag (ESA, Space Debris Office) for permission to use the MASTER population data.

\section{References}

Ananthasayanama, M.R., Anilkumarb, A.K., Subba Rao, P.V. A new stochastic impressionistic low Earth model of the space debris scenario, Acta Astronautica, 59 (7), 547-559, 2006.

Flohrer, T., Lemmens, S., Bastida Virgili, B., Krag, H., Klinkrad, H., Parrilla, E., Sanchez, N., Oliveira, J., Pina, F. DISCOS- current status and future developments, In: Proceedings of the Sixth European Conference on Space Debris, ESOC, Darmstadt, Germany, 22-25 April 2013, European Space Agency Publication SP-723, 2013.

Holling, C. S. Adaptive environmental assessment and management, John Wiley and Sons, Chichester, UK, 1978.

Johnson, N.L., Krisko P.H., Liou, J.C., Anz-Meadorc, P.D., NASA's New Break-up Model of Evolve 4.0, Advances in Space Research, 28 (9), 1377-1384, 2001.

Inter-Agency Space Debris Co-ordination Committee, IADC space debris mitigation guidelines, IADC-02-01, available at http://www.iadc-online.org (last accessed: 1/01/14), 2007.

Inter-Agency Space Debris Co-ordination Committee, Stability of the future LEO environment, IADC-12-08, available at http://www.iadc-online.org (last accessed: 1/01/14), 2013.

Kessler, D. J., Cour-Palais, B. G. Collision frequency of artificial satellites: the creation of a debris belt, Journal of Geophysical Research: Space Physics, 83 (A6), 2637-2646, 1978.

Klinkrad, H., Johnson, N.L. (Eds.), Space debris environment remediation, IAA Cosmic Study. International Academy of Astronautics, Paris, France, 2010.

Lewis, H.G., Swinerd, G.G., Newland, R.J., Saunders, A. Active removal study for on-orbit debris using DAMAGE, In: Proceedings of the Fifth European Conference on Space Debris, Darmstadt, Germany, 30 March-2 April 2009, European Space Agency Publication SP-672., 2009.

Lewis, H.G., Swinerd, G.G., Newland, R.J., Saunders, A. The fast debris evolution model, Adv. Space Res., 44 (5), 1865-1876, 2009. 
Lewis, H.G., Saunders, A., Swinerd, G.G, Newland, R.J. Effect of thermospheric contraction on remediation of the near-Earth space debris environment, Journal of Geophysical Research, 116, p. A00H08, doi:10.1029/2011JA016482, 2011.

Lewis, H.G., White, A.E., Crowther, R., Stokes, H. Synergy of debris mitigation and removal, Acta Astronautica, 81 (1), 62-68, 2012.

Liou, J.-C., Johnson, N.L. Risks in space from orbiting debris. Science, 311, 340-341, 2006.

Liou, J.-C., Johnson, N.L. A sensitivity study of the effectiveness of active debris removal in LEO, Acta Astronauta, 64 (2-3), 236-243, 2009.

Liou, J.-C., Johnson, N.L., Hill, N.M. Controlling the growth of future LEO debris populations with active debris removal. Acta Astronautica, 66 (5-6), 648-653, 2010.

National Oceanic and Atmospheric Administration National Geophysical Data Centre, available at http://www.swpc.noaa.gov/ (last accessed: 1/01/14), 2013.

Nazarenko, A.I. Prediction and analysis of orbital debris environment evolution, In: Proceedings of the First European Conference on Space Debris, Darmstadt, Germany, 5-7 April 1993, European Space Agency Publication ESA SD-Ol, 1993.

Nyberg, B. An introductory guide to adaptive management: for project leaders and participants, British Columbia Forest Service, Victoria, Canada, 1999.

Rex, D., Eichler, P. The possible long term overcrowding of LEO and the necessity and the effectiveness of debris mitigation measures, In: Proceedings of the First European Conference on Space Debris, Darmstadt, Germany, 5-7 April 1993, European Space Agency Publication ESA SD-Ol, 1993.

Reynolds, R.C., Fischer, N. H. Edgecombe D. S., A model for the evolution of the on-orbit man-made debris environment, Acta Astronautica, 10 (7), 479-498, 1983.

Rossi, A., Cordlli, A., Pardini, C., Anselmo, L., Farinella, P. Modelling the space debris evolution: two new computer codes, Advances in the Astronautical Sciences, Spaceflight Mechanics 1995, 1217-1231, AAS Publication, 1995.

Talent, D. Analytic model for orbital debris environmental management, Journal of Spacecraft and Rockets, 29 (4), 508-513, 1992.

Walters, C.J. Adaptive management of renewable resources, McGraw-Hill, New York, 1986. 
White, A., Lewis, H., The many futures of active debris removal. Acta Astronautica, 95, 189-197, 2014. 